\title{
Domain behavior in functional materials studied using Lorentz microscopy
}

\author{
C. Phatak ${ }^{1}$, S. Zhang ${ }^{1}$, W. Jiang ${ }^{1}$, S. G. E. te Velthuis ${ }^{1}$, A. Hoffmann ${ }^{1}$, J. F. Mitchell ${ }^{1}$, H. Zheng ${ }^{1}$, M. \\ R. Norman ${ }^{1}$, A. Petford-Long ${ }^{1}$ \\ ${ }^{1}$ Materials Science Division, Argonne National Laboratory, Lemont, IL 60439, USA
}

Lorentz tranmission electron microscopy (LTEM) is ideally suited for quantitative analysis of magnetic domains at the nanometer length scale. The ability to study both the microstructure and magnetic domain structure simultaneously in functional materials allows for direct understanding of the fundamental role of inhomogeneities in microstructure as well as the effect of shape and size of nanostructures on the magnetic domain behavior. The current state of art LTEM enabled using aberration correctors allows for imaging down to sub-nanometer scale in field-free conditions. In this talk, we will present the application of aberration corrected LTEM to study the magnetic domain behavior in various functional materials systems.

Understanding the underlying mechanism and phenomenology of colossal magnetoresistance (CMR) in layered manganites is essential from both a fundamental physics as well as from applications context. One of the proposed mechanisms for the CMR effect is that small ferromagnetic regions are formed which are connected in a percolative manner as the material undergoes a phase transition [1]. We have explored the in-situ phase transition from room temperature to below the Curie temperature of $T_{c}=125 \mathrm{~K}$ for bilayer manganite with a nominal composition of $\mathrm{La}_{1.24} \mathrm{Sr}_{1.76} \mathrm{Mn}_{2} \mathrm{O}_{7}$ [2]. We will show that from quantitative analysis of domain images parallel and perpendicular to the $\langle 001\rangle$ anisotropy axis (Figure 1(a) and (b)) enabled us to estimate the exchange stiffness, $\mathrm{A}=15.2 \mathrm{pJ} / \mathrm{m}$ from the domain wall width and to show that domain walls are tilted with respect to $\langle 001\rangle$. We will furthermore show that during the phase transition, direct real-space evidence for the formation of ferromagnetic nanoclusters close to $T_{c}$ was observed (Figure 1(c)), which coalesce as $\mathrm{T}$ is decreased further to form magnetic domains, indicating a percolative transition.

Topologically non-trivial spin textures such as skyrmions [3,4] present unique opportunities to explore exciting fundamental phenomena such as topological Hall effect as well as novel applications such as skyrmion-based spintronics. However, room-temperature realization of magnetic skyrmions has been a challenging topic, and recent work has shown that it is possible to form these structures in heterostructures with broken interfacial inversion symmetry [5]. We will present application of LTEM to visualize and understand the magnetic spin textures in two types of systems that can host skyrmions: (1) artificially patterned $\mathrm{Co} / \mathrm{Pt}$ films and (2) $\left[(\mathrm{Pt}(1.5) / \mathrm{Co}(1) / \mathrm{Mn}(1)]_{8}\right.$ systems. In the first case, using controlled focused ion beam irradiation, we will demonstrate the realization of artificial skyrmion and anti-skyrmion spin textures (Figure 2). Furthermore, using in-situ magnetizing experiments, we will show the effect of local anisotropy variation due to FIB patterning direction on the magnetization reversal. In the second case, using LTEM, we will elucidate the nature of the skyrmions to be either Bloch-type or Néel-type. We will also show the effect of applying pulsed electric current on the domain wall behavior in these systems.

References:

[1] M. Uehara, S. Mori, C. Chen, and S. Cheong, Nature, 399, 560 (1999). 
[2] C. Phatak, et. al., Phys. Rev. B, 92, 224418 (2015).

[3] X. Z. Yu, Y. Onose, N. Kanazawa, J. H. Park, J. H. Han, Y. Matsui, N. Nagaosa, and Y. Tokura, Nature, 465, 901 (2010).

[4] A. Fert, V. Cros, and J. Sampaio, Nat. Nanotechnol. 8, 152 (2013).

[5] W. Jiang et. al., Science, 349, 283 (2015).

[6] This work was supported by U.S. Department of Energy (DOE), Office of Science, Basic Energy Science, Materials Sciences and Engineering Division.
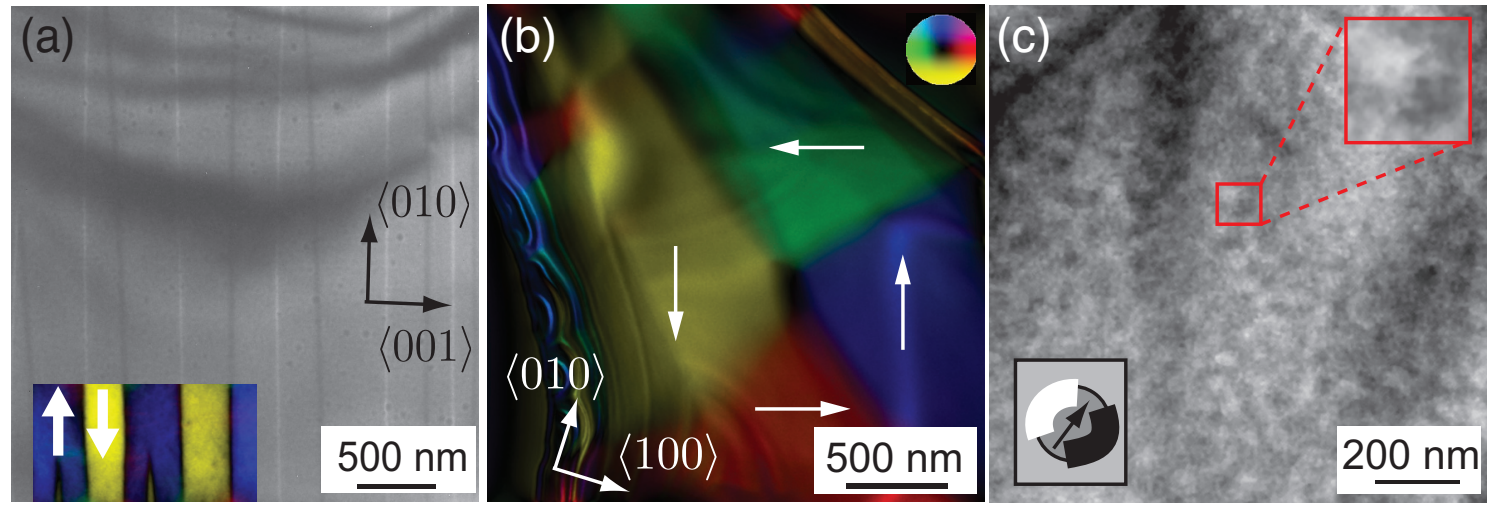

Figure 1: LTEM image and color magnetization maps along (a) $\langle 100\rangle$ and (b) $\langle 001\rangle$ showing strong magnetocrystalline anisotropy with a hard axis along $\langle 001\rangle$. (c) Ferromagnetic nanoscale clusters observed at the phase transition during in-situ cooling. Inset: high magnification image and schematic of a cluster (arrow shows magnetization direction)
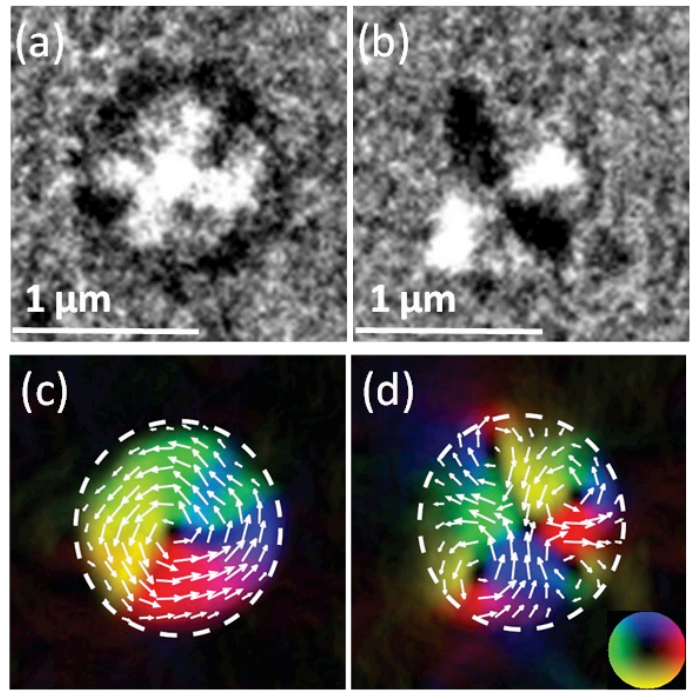

Figure 2: Under-focus LTEM images of ion irradiated discs of $1 \mu \mathrm{m}$ on $\mathrm{Co} / \mathrm{Pt}$ multilayer that form an artificial skyrmion(a) and antiskyrmion(b). Phase-reconstructed magnetization color maps are shown in (c) and (d) with arrows representing the in-plane magnetization directions, which are also indicated by the color wheel. Black contrast (c) and (d) indicates out-of-plane magnetization. 